\title{
Light-Driven Permanent Charge Separation across a Hybrid Zero-Dimensional/Two- Dimensional Interface
}

\begin{abstract}
Authors: Ilka Kriegel, Michele Ghini, Sebastiano Bellani, Kehao Zhang, Adam W. Jansons, Brandon M. Crockett, Kristopher M. Koskela, Edward S. Barnard, Erika Penzo, James E. Hutchison, Joshua A. Robinson, Liberato Manna, Nicholas J. Borys, P. James Schuck
\end{abstract}

This document is the unedited Author's version of a Submitted Work that was subsequently accepted for publication in The Journal of Physical Chemistry C, copyright (c) American Chemical Society after peer review. To access the final edited and published work see https:// doi.org/10.1021/acs.jpcc.0c01147

Kriegel, Ilka, Michele Ghini, Sebastiano Bellani, Kehao Zhang, Adam W. Jansons, Brandon M. Crockett, Kristopher M. Koskela, et al. "Light-Driven Permanent Charge Separation Across a Hybrid Zero-Dimensional/Two-Dimensional Interface." The Journal of Physical Chemistry C 124, no. 14 (March 16, 2020): 8000-8007. doi:10.1021/acs.jpcc.0c01147. 


\section{Light-Driven Permanent Charge Separation Across a Hybrid 0D-2D Interface}

Ilka Kriegel, ${ }^{a, b, c^{*}}$ Michele Ghini, ${ }^{a, d}$ Sebastiano Bellani, ${ }^{e}$ Kehao Zhang, ${ }^{f, g}$ Adam W. Jansons, ${ }^{h}$ Brandon M. Crockett, ${ }^{h}$ Kristopher M. Koskela, ${ }^{h}$ Edward S. Barnard, ${ }^{b}$ Erika Penzo, ${ }^{b}$ James E. Hutchison, ${ }^{h}$ Joshua A. Robinson, ${ }^{f, g}$ Liberato Manna, ${ }^{a}$ Nicholas J. Borys, ${ }^{b, i^{*}}$, P. James Schuck ${ }^{*}$

aDepartment of Nanochemistry, Istituto Italiano di Tecnologia, via Morego 30, 16163 Genova, Italy

${ }^{\mathrm{b}}$ The Molecular Foundry, Lawrence Berkeley National Laboratory, Berkeley, CA, USA

${ }^{\mathrm{c}}$ Department of Mechanical Engineering, Columbia University, New York, NY, USA

${ }^{\mathrm{d} D i p a r t i m e n t o ~ d i ~ C h i m i c a ~ e ~ C h i m i c a ~ I n d u s t r i a l e, ~ U n i v e r s i t a ̀ ~ d e g l i ~ S t u d i ~ d i ~ G e n o v a, ~ V i a ~}$ Dodecaneso 31, 16146 Genova, Italy

${ }^{\text {e}}$ Graphene Labs, Istituto Italiano di Tecnologia, via Morego 30, 16163, Genova, Italy fDepartment of Materials Science and Engineering, Center for 2-Dimensional and Layered Materials, The Pennsylvania State University, University Park, Pennsylvania, 16802, United States

gCenter for Atomically Thin Multifunctional Coatings (ATOMIC), The Pennsylvania State University, University Park, Pennsylvania, 16802, United States

hMaterials Science Institute and Department of Chemistry, University of Oregon, Eugene, Oregon 97403, United States

iDepartment of Physics, Montana State University, Bozeman, MT, USA

Corresponding Author*ilka.kriegel@iit.it, nicholas.borys@montana.edu, p.j.schuck@columbia.edu 


\begin{abstract}
.
We report the first demonstration of light-driven permanent charge separation across an ultrathin solid-state $0 \mathrm{D}-2 \mathrm{D}$ hybrid interface by coupling photoactive $\mathrm{Sn}$-doped $\mathrm{In}_{2} \mathrm{O}_{3}$ nanocrystals with monolayer $\mathrm{MoS}_{2}$, the latter serving as a hole collector. We demonstrate that the nanocrystals in this architecture act as local light-controlled charge sources by quasi-permanently donating $\sim 5$ holes per nanocrystal to the monolayer $\mathrm{MoS}_{2}$. The amount of photo-induced, contactless, charge-transfer to the monolayer $\mathrm{MoS}_{2}$ competes with what is reached in electrostatically gated devices. Thus, we have constructed a hybrid bilayer structure, in which the electrons and holes are separated into in two different solid-state materials. The temporal evolution of the local doping levels of the monolayer $\mathrm{MoS}_{2}$ follows a capacitor-like model with effective total capacitances in the femtofarad regime and areal capacitances in $\mu \mathrm{F} \mathrm{cm} ~^{-2}$ range. This analysis indicates that the $0 \mathrm{D}-2 \mathrm{D}$ hybrid system may be able to store light energy at densities of at least $\mu \mathrm{J} \mathrm{cm}^{-2}$, presenting new potential foundational building blocks for next-generation nanodevices that can remotely-control local charge density, power miniaturized circuitry, as well as harvest and store optical energy.
\end{abstract}




\section{INTRODUCTION.}

Portable, implantable, and wearable electronics with applications that range from medical diagnostics to retail consumer goods demand fundamentally new approaches for miniaturized architectures that can store and harvest energy. ${ }^{1-4}$ Achieving energetic self-sufficiency of micro- (and nano-) electronics is a major challenge towards realizing "energy autonomy" over extended periods. ${ }^{5,6}$ These goals could be met by a new class of nanosystems that break from the conventional paradigm of including two separate systems for energy conversion and storage. Multifunctional monolithic devices that incorporate charging and storage into the same system have the potential to fill this critical technology gap..$^{5}$

Solution-processed zero dimensional (0D) nanocrystals of metal oxides, such as doped indium oxide $\left(\mathrm{In}_{2} \mathrm{O}_{3}\right)^{7}$ or doped zinc oxide $(\mathrm{ZnO}),{ }^{8-10}$ offer an attractive suite of optoelectronic properties for such monolithic nanoscale energy platforms. They can sustain a high level of photodoping $\left(\sim 10^{21} \mathrm{~cm}^{-3}\right)$ and are capable of storing multiple extra electrons after photoexcitation. ${ }^{7-12}$ In these $0 \mathrm{D}$ systems, the requisite charge separation after above-gap excitation is readily achieved by efficient extraction of the hole from the nanocrystal by an external scavenging media. ${ }^{10,13,14}$ A single conductive metal oxide nanocrystal has been shown to store up to hundreds of electrons in liquid environments. ${ }^{8}$ Such "soluble supercapacitors" display volumetric capacitances of $\sim 233 \mathrm{~F} / \mathrm{cm}^{3}$, rivaling the performance of prototypical supercapacitor materials. ${ }^{8}$ To date, this remarkable light-driven charging capability has only been shown in systems where the hole scavenging media is a solution. However, for efficient energy-storage applications, an analogous nanoscale all solid-state architecture is needed.

In this work, we demonstrate that a prototype architecture of the two-dimensional (2D) semiconductor monolayer $\mathrm{MoS}_{2}\left(1 \mathrm{~L}-\mathrm{MoS}_{2}\right)$ covered by a layer of Sn-doped $\mathrm{In}_{2} \mathrm{O}_{3}$ (ITO) nanocrystals quasipermanently harvests and stores photoexcited carriers. The ITO nanocrystals serve as light-driven charge sources that transfer photogenerated holes into the $1 \mathrm{~L}-\mathrm{MoS}_{2}$, which serves as a solid-state hole scavenger. The charge transfer is stable for at least 72 days, a timeframe not achieved in other photodoping modalities. ${ }^{15-17}$ Corresponding carrier density variations in the $1 \mathrm{~L}-\mathrm{MoS}_{2}$ of $\sim 10^{13} \mathrm{~cm}^{-2}$ are observed and are comparable to the doping levels attained with electrostatic devices. ${ }^{18-20}$ The charge-transfer leaves $\sim 5$ electrons per single ITO nanocrystal, which are subsequently stored. From the standpoint of the ITO nanocrystals, these solid-state photodoping levels approach those of ITO and similar nanocrystals in solution. ${ }^{8}$ Unlike previous works, here we provide strong evidence that the hole is captured and stored in the $2 \mathrm{D}$ material rather than lost by reacting with a hole scavenger in solution. Thus, we were able to translate the established liquid-based supercapacitor approach to a hybrid bilayer system in which the electrons and holes are captured in two different layers of a solid state architecture, eliminating the need for a liquid environment. The kinetic behavior of the system exhibits capacitor-like charging with effective characteristic capacitances in the femtofarad (fF) regime, corresponding to volumetric and areal capacitances of $\sim 1 \mathrm{~F} / \mathrm{cm}^{3}$ and $\sim 1 \mu \mathrm{F} / \mathrm{cm}^{2}$, respectively. This 0D-2D hybrid system reveals novel potential approaches for light-driven nanoscale energy storage as well as wireless, optically controlled doping of 2D semiconductors over a broad range of carrier concentrations. 


\section{METHODS.}

\section{Nanocrystal synthesis and characterization}

$\mathrm{ITO} / \mathrm{In}_{2} \mathrm{O}_{3}$ core/shell nanocrystals were synthesized in a manner similar to that described in literature. ${ }^{36,37}$ Two precursor solutions were prepared in separate vials. In one vial, tin(IV) acetate and indium(III) acetate were mixed in a 1:9 Sn:In ratio. Oleic acid was added to this vial in a 1:6 metal to acid ratio to yield a $10 \% \mathrm{Sn}$ doped ITO precursor solution. In a separate vial, indium(III) acetate was mixed with oleic acid in a 1:6 molar ratio to yield an undoped indium oleate precursor solution. Both vials were left at $150{ }^{\circ} \mathrm{C}$ under $\mathrm{N}_{2}$ for several hours. The ITO nanocrystal cores were first prepared by adding the ITO precursor solution dropwise (at a rate of 0.35 $\mathrm{mL} \mathrm{min}^{-1}$ ) via a syringe pump to $13.0 \mathrm{~mL}$ of oleyl alcohol at $290{ }^{\circ} \mathrm{C}$. The ITO nanocrystal cores were grown to a size of $5.9 \mathrm{~nm}$, and a small aliquot was taken out of the reaction mixture for analysis. After the addition of the ITO precursor solution, the undoped indium oleate precursor solution was added in a similar manner as described above. Enough undoped indium oleate precursor was added to allow the nanocrystals to grow to a final size of $10.1 \mathrm{~nm}$. Nanocrystals were isolated by precipitating with $\sim 12 \mathrm{~mL}$ ethanol. The solid was collected by centrifugation at $7000 \mathrm{rpm}$ for $10 \mathrm{~min}$. The solid was then collected and washed twice more with ethanol.

Nanocrystals were characterized for size and polydispersity by small-angle X-ray scattering (SAXS) and transmission electron microscopy (TEM). SAXS analysis was done on a lab-scale SAXS (SAXSess, Anton Par, Austria) equipped with an X-ray tube $(\mathrm{Cu} \mathrm{K \alpha})$ operating at $40 \mathrm{kV}$ and $50 \mathrm{~mA}$. The scattered X-ray intensities were measured with a charge-couple device detector (Roper Scientific, Germany). Raw data was processed with SAXSquant software (version 2.0). Scattering curves were averaged over 50 individual curves for various acquisition times (0.2-10s). Curve fitting was done using Irena macros for IGOR (V. 6.3.7.2). ${ }^{21}$ TEM analysis was implemented to corroborate SAXS analysis, and was done using an FEI Tecnai Spirit TEM (Hillsboro, OR) operating at $120 \mathrm{kV}$. Nanocrystals were imaged on Ted Pella (Redding, CA) lacey carbon grids supported by a copper mesh.

\section{$\mathrm{MoS}_{2}$ sample preparation}

The $\mathrm{MoS}_{2}$ monolayer film is prepared by powder vaporization technique similar to our previous work. ${ }^{22} 2 \mathrm{mg}$ $\mathrm{MoO}_{3}$ powder (Sigma Aldrich, 99.97\%) and $200 \mathrm{mg}$ sulfur powder (Sigma Aldrich, 99.998\%) is located in two hot zones in a home-built horizontal furnace (2"' tube diameter). Prior to the growth, the chamber is pumped to the base pressure ( $18 \mathrm{mTorr}$ ) for $5 \mathrm{~min}$ to remove the moisture and contaminations. During the growth, the $\mathrm{MoO}_{3}$ along with the substrate is heated up to $550{ }^{\circ} \mathrm{C}$ for $2 \mathrm{~min}$ for the nucleation, followed by tuning the temperature for $\mathrm{S}$ powder to $130^{\circ} \mathrm{C}$ when the nucleation step is finished. The chamber temperature is increased to $725^{\circ} \mathrm{C}$ for 15 min while keeping the $\mathrm{S}$ powder at $130{ }^{\circ} \mathrm{C} .100 \mathrm{sccm} \mathrm{Ar}$ is employed as the carrier gas in the growth.

\section{Hybrid sample formation}

Nanocrystal films were deposited over the monolayer $\mathrm{MoS}_{2}$ sample by spin coating $20 \mu \mathrm{L}$ of the $10 \mathrm{mg} \mathrm{mL}^{-1}$ stock solution for $45 \mathrm{sec}$ and $2000 \mathrm{rpm}$. A thin layer of poly(methyl methacrylate) was spin-coated by depositing $50 \mu \mathrm{L}$ of a $5 \mathrm{mg} \mathrm{mL}^{-1}$ stock solution for $45 \mathrm{sec}$ and $2000 \mathrm{rpm}$.

\section{Optical measurements}

PL imaging was performed on a scanning confocal microscope implementing pulsed laser excitation $(\lambda=500 \mathrm{~nm} ; 80 \mathrm{MHz}$ repetition rate) focusing onto the sample with a $50 \times 0.6$ NA objective to a diffractionlimited spot. PL from the sample was collected by the same objective, and reflected laser light was filtered from the collected light using a $550 \mathrm{~nm}$ long-pass filter combined with a $532 \mathrm{~nm}$ long-pass dichroic mirror. The filtered PL dispersed by a spectrometer (Princeton Instruments) and detected with a cooled charge coupled device (CCD) camera (Andor). Mapping was performed by raster-scanning the sample with high precision piezo stages (Mad City Labs) and collecting PL spectrum at each spatial position using custom microscopy software. ${ }^{23}$ For all excitation energies, the excitation density was kept in the range in which the PL scales 
linearly with excitation density. Optical photodoping was obtained by focusing a $355 \mathrm{~nm}$ pulsed laser onto a spot of the sample for a specific time interval and collecting the PL spectra in a continuous way.

\section{RESULTS AND DISCUSSION}

a)

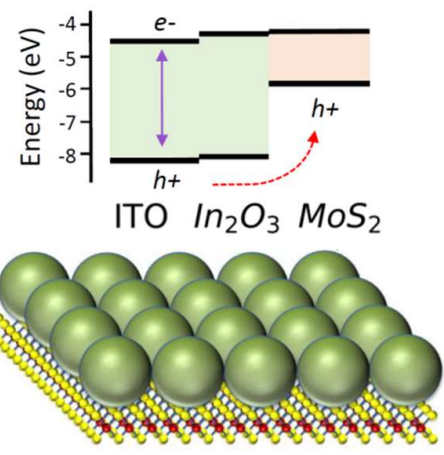

c)

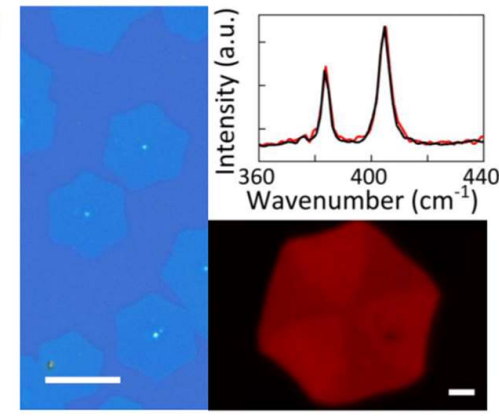

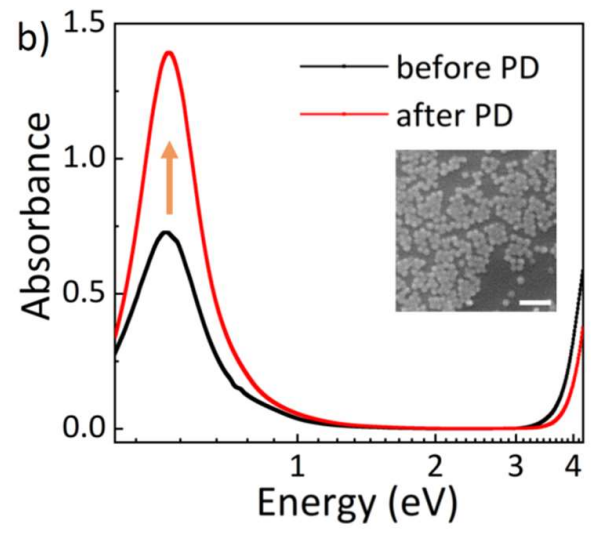

d)

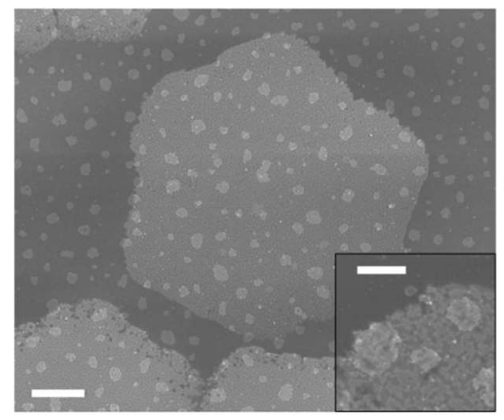

\section{Photo-induced electron extraction from 1L-MoS2}

Figure 1 - Hybrid nanostructure of ITO nanocrystals and 1-ML MoS $\mathbf{S}_{2}$. a) Illustration of the hybrid system of ITO nanocrystals (green spheres) deposited over $1 \mathrm{~L}-; \mathrm{MoS}_{2}$ together with the approximate band alignment. Photoexcited holes inside the ITO nanocrystals (e.g. generated by optical excitation with photon energies in excess of the bandgap of ITO) are rapidly transferred to the 2D material, while photo-electrons accumulate in the nanocrystal. b) Optical absorbance spectrum of the ITO nanocrystals in solution, with the typical localized surface plasmon resonance in the near IR before (black line) and after (red line) photodoping in solution. Inset shows a SEM image of the nanocrystals. Scale bar is $50 \mathrm{~nm}$, the average dimension of a single nanocrystals is $\sim 10 \mathrm{~nm}$. c) Typical optical micrograph (scale bar $=15 \mu \mathrm{m}$ ) and micro-PL measurement (scale bar $=2 \mu \mathrm{m}$ ) of the CVD grown $1 \mathrm{~L}-\mathrm{MoS}_{2}$ sample. On the top-right: Raman spectroscopy of a flake without (black line) and with (red line) the nanocrystal layer, the in-plane ( $\left.E_{2 g}\right)$ mode and the out-of-plane ( $\mathrm{A}_{1 \mathrm{~g}}$ ) mode located respectively around $\sim 383 \mathrm{~cm}^{-1}$ and $\sim 404 \mathrm{~cm}^{-1}$. d) Typical SEM image of the hybrid nanostructure, with two-dimensional flakes of $1 \mathrm{~L}-\mathrm{MoS}_{2}$ covered with a thin film of ITO nanocrystals. Scale bar $=1 \mu \mathrm{m}$, inset scale bar $=500 \mathrm{~nm}$.

Figure 1 shows the hybrid 0D-2D nanosystem studied here. The proof-of-concept system is fabricated by spincasting ITO nanocrystals on crystallites of $1 \mathrm{~L}-M o S_{2}$, as illustrated in Figure 1a. The ITO nanocrystals are composed of a Sn-doped $\mathrm{In}_{2} \mathrm{O}_{3}$ core with a surface protection layer $\left(\sim 1.25 \mathrm{~nm}\right.$ thick) of undoped $\mathrm{In}_{2} \mathrm{O}_{3} .{ }^{24-26}$ The doping is confirmed by the presence of a strong absorption peak around $2500 \mathrm{~nm}$ as seen in the absorbance spectrum in Figure 1b, which arises from the localized surface plasmon resonance due to the free carrier density of the small spherical nanocrystals. ${ }^{12}$ The absorption onset in the ultraviolet (UV) spectral range at $355 \mathrm{~nm}$ corresponds to the bandgap of the ITO nanocrystals. The effect of exposure of ITO nanocrystals in solution to UV light beyond their bandgap is shown in Figure $1 \mathrm{~b}$ and Figure S1a of the Supporting Information and manifests itself as a shift and intensity enhancement of the plasmon resonance due to the presence of additional photodoped carriers in the nanocrystals. ${ }^{7,8,12,13}$ This effect is stable as long as the system is stored in an inert atmosphere. The $1 \mathrm{~L}-\mathrm{MoS}_{2}$ flakes are grown on $\mathrm{SiO}_{2} / \mathrm{Si}$ substrate using conventional CVD-growth. The lateral 
dimensions of the $1 \mathrm{~L}-\mathrm{MoS}_{2}$ crystallites are $\sim 15 \mu \mathrm{m}$ (see Figure 1c). The monolayer nature is confirmed by Raman measurements, displaying the in-plane $\left(\mathrm{E}_{2 \mathrm{~g}}\right)$ mode and the out-of-plane $\left(\mathrm{A}_{1 \mathrm{~g}}\right)$ mode located respectively around $\sim 383 \mathrm{~cm}^{-1}$ and $\sim 404 \mathrm{~cm}^{-1}$. Finally, a thin polymer encapsulation layer is spin-cast on the sample to mitigate the effects of exposure to ambient atmosphere. A scanning electron micrograph image that shows nearly complete coverage of the $1 \mathrm{~L}-\mathrm{MoS}_{2}$ with the ITO nanocrystals decorated with isolated islands of thicker coverage is shown in Figure 1d. Complete synthesis details are provided in the Methods section.

As shown in Figure 2, photoluminescence (PL) spectroscopy is used to monitor changes in the carrier density of the $1 \mathrm{~L}-\mathrm{MoS}_{2}$. In 1L-transition metal dichalcogenides (TMDCs) the relative populations of tightly bound excitons and trions at room temperature serves a non-invasive probe of carrier density. ${ }^{18,19,27,28} \mathrm{~A}$ trion is an exciton that is bound to an extra free carrier, which is stable in the 2D semiconductor at room temperature as a result of the greatly enhanced Coulomb interactions. ${ }^{18}$ The amount of trions and thus the intensity of their photoluminescence depends on the density of free carriers in the system, and relative changes in carrier density can be probed via the photoluminescence spectrum, circumventing the need to make electrical connections with bulky contacts. Several recent works have displayed the usefulness of photoluminescence spectroscopy as a tool to non-invasively extract carrier density variations in 2D TMDCs. ${ }^{18,19,27,28}$ We first characterized the hybrid system without photoexciting the ITO nanocrystals by selectively exciting only the $1 \mathrm{~L}-\mathrm{MoS}_{2}$ with 2.48 $\mathrm{eV}\left(500 \mathrm{~nm}\right.$ ) optical excitation. Under these conditions, the $1 \mathrm{~L}-\mathrm{MoS}_{2} \mathrm{PL}$ (red curve in the top panel of Figure 2b) is centered around $1.81 \mathrm{eV}$ and is composed of two distinct bands: a high-energy band from neutral excitons (blue component) and a low-energy band from trions (green component). The emission intensity of the low-energy trion relative to the neutral exciton, as well as their energies and energetic separations are acutely sensitive to the density of free carriers in the $1 \mathrm{~L}-\mathrm{MoS}_{2} \cdot{ }^{18,19,27,28}$ The initial strong presence of the trion and the low-energy of the PL indicate that $1 \mathrm{~L}-\mathrm{MoS}_{2}$ is substantially n-doped in the hybrid architecture prior to the photodoping process.

To study the photodoping process (i.e., p-type photodoping due to the transfer of holes from the ITO nanocrystals into the $\left.1 \mathrm{~L}-\mathrm{MoS}_{2}\right)$ the hybrid system was optically excited with UV light at $3.54 \mathrm{eV}(355 \mathrm{~nm}$; see additional details in the Methods section). This energy is above the bandgaps of both the $1 \mathrm{~L}-\mathrm{MoS}_{2}$ and the ITO nanocrystals, enabling the monitoring of the $1 \mathrm{~L}-\mathrm{MoS}_{2} \mathrm{PL}$ while photoexciting the nanocrystals. The temporal evolution of the PL of the $1 \mathrm{~L}-\mathrm{MoS}_{2}$ in the hybrid system under this UV excitation is shown in Figure 2a by plotting the evolution of the spectral median with time of photodoping. The spectral median is defined as the energy that divides the spectrum into equal amounts of high and low-energy emission. This quantity is a convenient descriptor of the data that captures both peak shifts and changes in lineshape. An immediate blue shift of the PL of the $1 \mathrm{~L}-\mathrm{MoS}_{2}$ is observed (purple, $0 \mathrm{~min}$ ) by $15.9 \mathrm{meV}$. This trend continues as the PL of the $1 \mathrm{~L}-\mathrm{MoS}_{2}$ progressively shifts to higher energies over the course of $\sim 10$ minutes after which no further changes occur, reaching a maximum shift of $29.4 \mathrm{meV}$. Remarkably, the photodoping and changes to the $1 \mathrm{~L}-\mathrm{MoS}_{2}$ persist long-after the photodoping is terminated, as was observed by re-measuring the $1 \mathrm{~L}-\mathrm{MoS}_{2} \mathrm{PL}$ with 2.48 $\mathrm{eV}$ excitation after an additional 10 minutes (blue star in Figure 2a). Further experiments confirmed the irreversibility of the processes up to 72 days after the light-induced charge transfer, indicating that the photodoping process is permanent (see Figure S2 of the Supporting Information). Importantly, in control measurements of the $1 \mathrm{~L}-\mathrm{MoS}_{2}$ without the ITO nanocrystals, the PL spectrum does not evolve with time under the same illumination conditions (Figure S1b of the Supporting Information), highlighting that the ITO nanocrystals play a crucial role in the charging process reported here. 


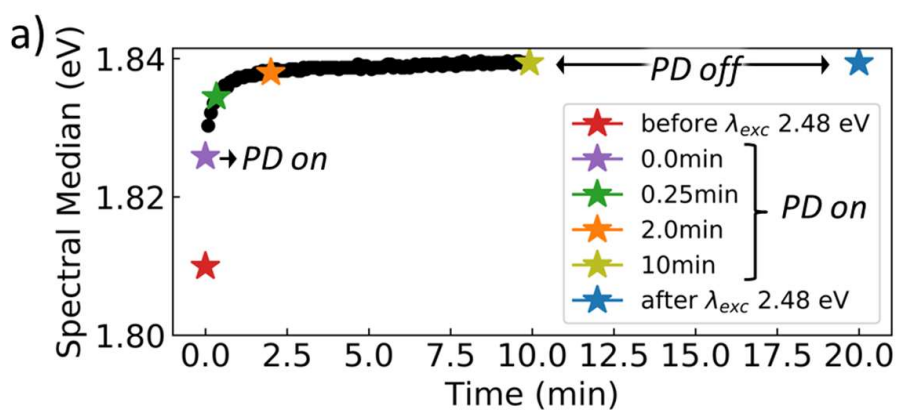

b)

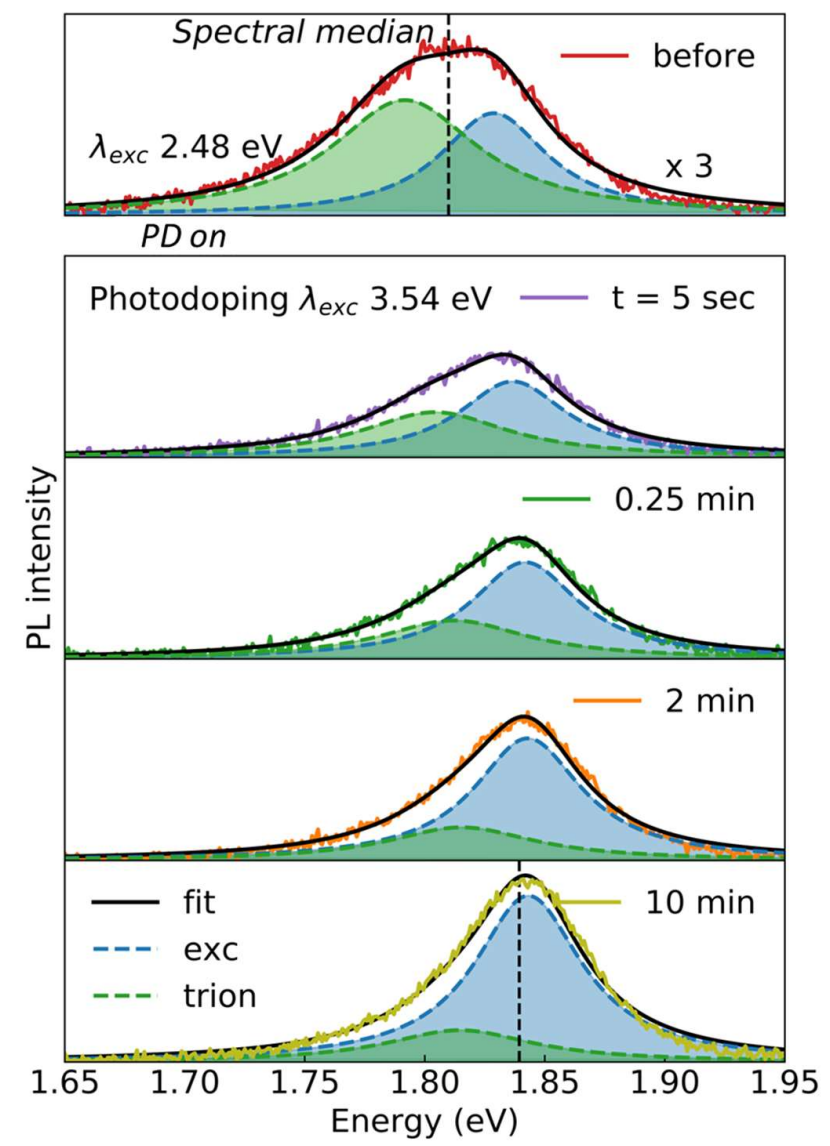

Figure 2 - Temporal evolution of the spectrum of the $1 \mathrm{~L}-\mathrm{MoS}_{2} \mathrm{PL}$ in the 0D-2D hybrid nanosystem during photo-induced hole extraction (p-photodoping). a) Spectral median shift and b) evolution of the PL spectra of the monolayer MoS2 with time of photodoping (PD). The stars in a) correspond to the same color-coded PL spectra in b). The initial PL spectrum of the $1 \mathrm{~L}-\mathrm{MoS} 2 \mathrm{with}$ an excitation energy of $2.48 \mathrm{eV}(500 \mathrm{~nm}$; no ITO excitation) is given in red. After photodoping with $3.54 \mathrm{eV}$ (355 nm; above the bandgap of both the 1L-MoS 2 and the ITO nanocrystals) an immediate blue shift to higher energy is observed (purple curve), which further blue shifts and intensifies with increasing exposure up to $10 \mathrm{~min}$ (compare green to yellow spectra). The PL spectra are fitted by the sum (black curves) of two Lorentzian line shapes, which are assigned to emission from the exciton (blue curves) and trion (green curves) states. The permanent variation of the PL was confirmed by re-measuring the $1 \mathrm{~L}-\mathrm{MoS} 2 \mathrm{PL}$ with $2.48 \mathrm{eV} 10 \mathrm{~min}$ after the photodoping was switched off (no ITO excitation, blue star in a).

The variation of the spectra with photodoping is illustrated in Figure 2b. By comparing the spectrum of the initial PL of the $1 \mathrm{~L}-\mathrm{MoS}_{2}$ (top panel, Figure b) to that of the final PL after 10 minutes of photodoping (bottom panel, Figure b), it is clear that the PL (1) increases in intensity, (2) shifts in energy, and (3) changes spectral line shape. These changes are identical to what is observed when the density of n-type free carriers is reduced in an electrostatically gated device ${ }^{18-20}$ or by locally inhomogeneous dielectric environments. ${ }^{29-31}$ Therefore, these changes in the hybrid system under photodoping are attributed to a substantial reduction in n-type carriers 
in the $1 \mathrm{~L}-\mathrm{MoS}_{2}$ due to a charge transfer from the ITO nanocrystals. As more free electrons are removed, the relative spectral weight of the trion decreases and the overall PL intensity increases, since the PL quantum yield of trions is lower than that of excitons. ${ }^{18,19}$ Notably, at $\mathrm{t}=0 \mathrm{~min}$ when the ITO nanocrystals are initially excited, a significant decrease of the relative weight of the trion state is immediately observed, indicating rapid and intense initial transfer of holes to the $1 \mathrm{~L}-\mathrm{MoS}_{2}$. Thereafter, the $1 \mathrm{~L}-\mathrm{MoS}_{2} \mathrm{PL}$ spectrum continuously evolves at a slower rate signifying that there are two different time constants for the photodoping process. The first rate is apparently faster than the temporal resolution of our experiment $(\sim 5 \mathrm{~s})$, whereas the second rate yields changes spanning several minutes.

\section{Temporal investigation of carrier extraction}

To more deeply analyze the photodoping dynamics of the hybrid structure, we recorded the PL spectrum of the $1 \mathrm{~L}-\mathrm{MoS}_{2}$ every 5 seconds over the course of a full 10 minutes of photodoping. The evolution of the exciton and trion populations and energies are shown in Figure 3. A significant reduction in the relative spectral weight of the trion and a corresponding increase in that of the exciton is observed, resulting in an increase of the exciton-to-trion ratio $\left(I_{\text {exc. }} / I_{\text {trion }}\right)$ from $\sim 0.67$ to $\sim 4.0$ over the photodoping process (Figure $3 \mathrm{a}$ ). We attribute this significant increase in the exciton-to-trion ratio to a reduction in the density of negatively charged carriers in the $1 \mathrm{~L}-\mathrm{MoS}_{2}$ due to photoinduced hole injection from the ITO nanocrystals. The change in the carrier density is corroborated by the substantial blue-shift of the energy of the exciton and trion ( $E_{\text {exc }}$ and $E_{\text {trion }}$, respectively; Figure $3 b$ ), which follow nearly identical dynamics. Further, the energetic separation between the exciton and trion ( $\Delta E=E_{\text {exc }}-E_{\text {trion }}$ ) decreases from $\sim 37 \mathrm{meV}$ to $\sim 28.5 \mathrm{meV}$ (Figure 3c). The final energetic separation achieved is $\sim 10 \mathrm{meV}$ above the anticipated trion binding energy of $18 \mathrm{meV}$ for intrinsically doped $1 \mathrm{~L}-\mathrm{MoS}_{2}{ }^{18}$ This indicates a slightly $\mathrm{n}$-doped character of the $1 \mathrm{~L}-\mathrm{MoS}_{2}$ after the photodoping process, which is consistent with the residual non-zero relative intensity of the trion.
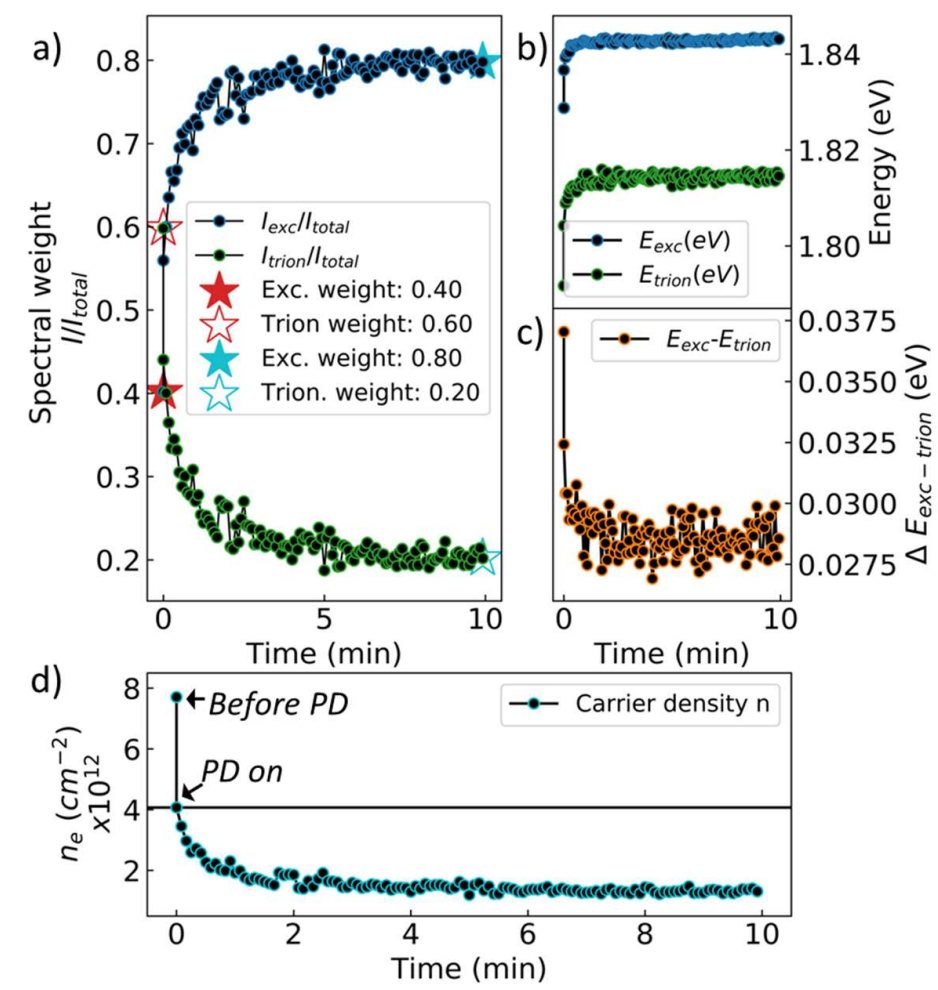

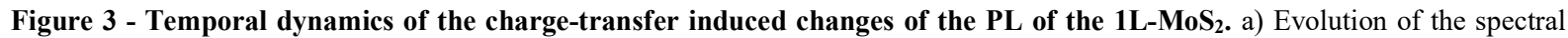
weights of the exciton (blue) and trion (green) normalized to the total spectrum showing an increase in excitons at the expense of trions during the photodoping. The evolution of $b$ ) the energies of the exciton and trion and $c$ ) their energetic separation over the duration of 
the photodoping. d) The dynamics of the total change of the carrier density as extracted from the evaluation of the ratio of the emission intensity of the exciton to that of the trion.

Notably, such deconvolution of room temperature PL into the exciton and trion components can be used to extract the level of doping in TMDCs. ${ }^{18,19,27,28}$ A modified mass action model that connects the free carrier density to the ratio between the spectral weight of the exciton and the trion is used to quantify the photoinduced changes of the carrier density in the $1 \mathrm{~L}-\mathrm{MoS}_{2}$. In more detail, for low excitation densities (i.e., when the density of excitons is much smaller than the density of free carriers), the thermal equilibrium between the populations of excitons $\left(n_{x}\right)$, trions $\left(n_{x}\right)$ and free carriers $\left(n_{e}\right)$ is given by a Boltzmann distribution. Such a model was originally used to describe the equilibrium of $n_{x}+n_{e} \leftrightarrow n_{x}$ - in conventional epitaxial quantum well structures to accurately predict the variation of the carrier density ${ }^{32,33}$ and has already been adapted to layered $2 \mathrm{D}$ materials (e.g. $1 \mathrm{~L}-\mathrm{MoS}_{2}$ and $1 \mathrm{~L}-\mathrm{MoSe}_{2}$ ) in order to estimate the exciton-to-trion ratio. ${ }^{19,27,28}$ Here, we similarly adapt it to describe the temporal evolution of the exciton-to-trion ratio in terms of the variation of the carrier density due to photodoping. Specifically, the evolution of carrier density, $n_{e}(t)$ is:

$$
n_{e}(t)=\frac{n_{x}-(t)}{n_{x}(t)} \cdot \frac{4 M_{X_{0}} m_{e}}{\pi \hbar^{2} M_{x^{-}}} \cdot k_{B} T \cdot e^{\left(-\frac{E_{B}^{x^{-}}}{k_{B} T}\right)},
$$

where $m_{e}$ is the effective mass of the electron, $M_{X 0}$ and $M_{x \text { - }}$ are the effective masses of the exciton and trion respectively, and $E_{B}^{x^{-}}$is the intrinsic trion binding energy (taken from Ref. ${ }^{18}$ ). As shown in Figure 3d, during the photodoping process, the carrier density $n_{e}$ decreases by $\sim 6 \times 10^{12} \mathrm{~cm}^{-2}$, which is in the range of free carrier changes achieved in electrostatic p-doping of $1 \mathrm{~L}-\mathrm{MoS}_{2} \cdot{ }^{18,34}$ Furthermore, the final free carrier density reached is approximately $1.5 \times 10^{12} \mathrm{~cm}^{-2}$, which, in comparison to the independent electrostatic p-doping measurements, ${ }^{18}$ agrees with the free carrier density needed to yield an exciton-to-trion ratio of 4.0 and an energetic separation between the exciton and trion of $28.5 \mathrm{meV}$ to which our system ultimately converges. ${ }^{18}$

\section{a) Nanocrystals}

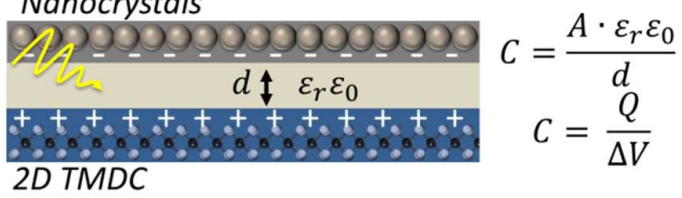

b)

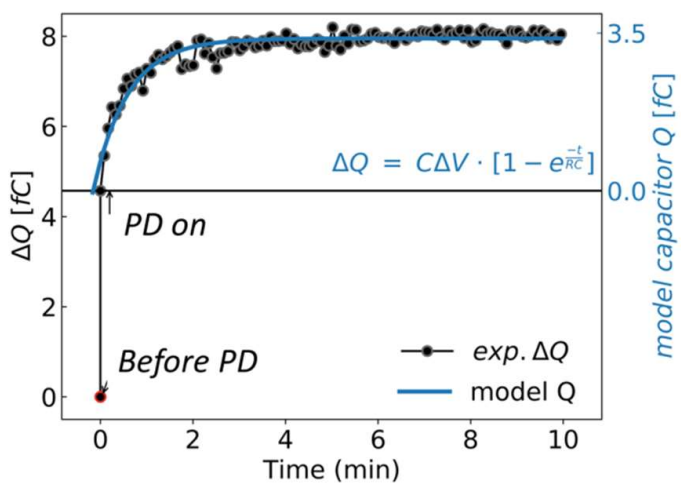

Figure 4 - Charge density variation, $\Delta Q$, in a capacitor model of the hybrid 0D-2D system. a) Illustration of the capacitor model for the light-driven charge separation across the $0 \mathrm{D}-2 \mathrm{D}$ hybrid interface. b) Charge density variation extracted from the analysis of exciton/trion PL ratio (black curve) together with the fit to the capacitor equation (blue curve).

\section{Approximating the hybrid 0D-2D system as a model capacitor.}

From the total amount of charges transferred within the excitation spot of the laser $(\sim 1 \mu \mathrm{m}$ in diameter) we can additionally provide an estimate for the average amount of carriers transferred per nanocrystal. Conservatively 
assuming complete coverage of the nanocrystals with diameters of $\sim 10.1 \mathrm{~nm}$ (in a closed-pack arrangement) within the excitation spot, we estimate that an average of 5 holes per nanocrystal are transferred to the localized region of $1 \mathrm{~L}-\mathrm{MoS}_{2}$ directly underneath the nanocrystals. This value is of the same order of magnitude of those observed for photodoped metal oxide nanocrystals in solution as compared to literature values. ${ }^{8,9}$

After light absorption in the 0D semiconductor nanocrystal film, the photogenerated charges are separated thanks to the efficient collection of holes by the 2D semiconductor (i.e., $1 \mathrm{~L}-\mathrm{MoS}_{2}$ ). The nanocrystal film becomes negatively charged and the $1 \mathrm{~L}-\mathrm{MoS}_{2}$ layer becomes positively charged, separated by the dielectric nanocrystal shell and ligands. In a recent work on 2D material hetero bilayers, photo-excited charges quickly form an electron-hole bilayer across a hetero-interface. The separated charges across the 2D material interface form the positive and negative electrodes of a capacitor. In this work, a simple capacitive model has been used to approximate the electronic behavior of this system during photoexcitation, despite relying on photoexcited populations that decay on ultrafast timescales. ${ }^{35}$ These transient effects are substantially different from our observations where quasi-permanent effects are observed. As shown in Figure 4a, we approximated our hybrid 0D-2D nanosystem with a basic model capacitor, consisting of two plate-like electrodes separated by a nonconductive region. Here, the film of $0 \mathrm{D}$ nanocrystals and the $2 \mathrm{D} 1 \mathrm{~L}-\mathrm{MoS}_{2}$ are the negative and positive electrodes, respectively. The electrodes are separated by a dielectric material of width $d$, which is determined by the shell thickness surrounding the cores of the $0 \mathrm{D}$ nanocrystals. This estimate is reasonable, considering that the ITO- $\operatorname{In}_{2} \mathrm{O}_{3}$ core-shell structure creates a heterostructure band alignment that localizes electrons to the core and holes to the surface of the nanocrystals from where they are transferred to the 2D material. We additionally take into account the added dielectric layer associated with the surface ligands. With a ligand length of $\sim 1.5 \mathrm{~nm}$, a ligand shell thickness of around $0.8 \mathrm{~nm}$ is expected. ${ }^{36}$ Thus, we estimate a total dielectric thickness of $d=2.05 \mathrm{~nm}$ (including the $\mathrm{In}_{2} \mathrm{O}_{3}$ shell of $1.25 \mathrm{~nm}$ ) and an effective refractive index $\varepsilon_{r} \approx 2$, which accounts for the ligands, the $\operatorname{In}_{2} \mathrm{O}_{3}$ shell, and the empty space which comprise the volume between the nanocrystal core and the $1 \mathrm{~L}-\mathrm{MoS}_{2}$ (see discussion and Figure S3 in the SI). The capacitance $(C)$ between the electrodes can be estimated as $C=\varepsilon_{r} \varepsilon_{0} \frac{A}{d}$ and subsequently, the voltage difference as $\Delta V=\frac{Q}{C}$.Using this model with the absolute charge density variation, $Q$, in the illumination area (i.e., the steady-state value of 8.0 $\mathrm{fC}$ reached in Figure $4 \mathrm{~b}$ ) and the effective refractive index $\varepsilon_{r} \approx 2$, the estimated capacitance of a nanocrystal$1 \mathrm{~L}-\mathrm{MoS}_{2}$ junction is $\sim 6.9 \mathrm{fF}$, which corresponds to an areal capacitance of $\sim 0.88 \mu \mathrm{F} \mathrm{cm}^{-2}$ (with the illumination area approximated as $0.78 \mu \mathrm{m}^{2}$ ). Furthermore, the estimated voltage difference between the nanocrystal film and $1 \mathrm{~L}-\mathrm{MoS}_{2}$ is $\sim 1.16 \mathrm{~V}$, which reasonably agrees with the anticipated potential difference of $1.27 \mathrm{eV}$ between the conduction band of the ITO nanocrystals (e.g. $-4.6 \mathrm{eV}$, taken from ref. ${ }^{37}$ ) and valence band of $1 \mathrm{~L}-\mathrm{MoS}_{2}$ (e.g. $-5.87 \mathrm{eV}$, taken from ref. ${ }^{38}$ ).

In addition to the steady state analysis, the temporal response of the system can also be fit to a basic capacitor model as shown in Figure 4b. Immediately after UV illumination, the temporal evolution of the charge density in the $1 \mathrm{~L}-\mathrm{MoS}_{2}$ strongly resembles the transient response of a capacitor, which can be fitted by: $Q=C \Delta V$. $\left[1-e^{-t / R C}\right]$. By assuming the previously estimated voltage difference (i.e., $\Delta V=1.16 \mathrm{~V}$ ), the capacitance extracted by fitting the dynamics is $2.3 \mathrm{fF}$. This value is smaller than the estimate provided above (i.e., $\sim 6.9$ fF) because the fit to the dynamics neglects the significant offset between the initial charge density and the charge density immediately after photodoping. This initial charge transfer from the ITO nanocrystals to the $1 \mathrm{~L}-\mathrm{MoS}_{2}$ is faster than the temporal-resolution of our measurement $(\sim 5 \mathrm{~s})$ but accounts for more than half of the total carriers transferred. The second, slower dynamics of the charging process, displays a capacitor charging time constant of $R C^{-1} \approx 45 s$ as extracted from the fit. These distinct timescales suggest that reactions between the excited ITO nanocrystals and surface-absorbed chemical species (e.g., an oxygen reduction reaction) as well as a depleted space charge region close to the nanocrystal surface ${ }^{39-42}$ may also play important roles in the photodoping process. Such a chemical process would lower the estimated voltage difference (i.e., $\Delta V$ ) as it would originate from the potential energy of the involved chemical reaction, rather 
than the potential difference due to the band offsets between the ITO nanocrystals and $1 \mathrm{~L}-\mathrm{MoS}_{2}$. Nevertheless, these additional potential contributions highlight that the particular treatment and composition of the nanocrystal surface can be utilized to modify the capacitive properties of the hybrid structure. This simple prototypical light-driven, ultrathin 0D-2D hybrid capacitor with an overall thickness of $\sim 12 \mathrm{~nm}$ is estimated to store energy densities in the range of $1 \mu \mathrm{J} \mathrm{cm}^{-2}$ over quasi-permanent timescales. Considering the two timescales, this stored energy equates to power densities of $\mathrm{nW} \mathrm{cm}$ - for the slower charging process and $\mu \mathrm{W}$ $\mathrm{cm}^{-2}$ for the faster charging process (considering that the resolution limit of this measurement is $5 \mathrm{~s}$ ). Notably, the latter of these power densities approaches typical values of 2D material based supercapacitor platforms. ${ }^{4}$ By optimizing the nanomaterial constituents, which span a wide range of possible 0D nanocrystals and 2D materials, and exploring more sophisticated geometries which enhance the active capacitor area, we anticipate that substantially improved performance can be achieved. Currently, investigations of the reversibility of the charging process and the exploitation of the stored carriers in a capacitor device is still on-going. However, we see potential for this hybrid structure as a great step forward towards the light-driven energy storage.

\section{CONCLUSIONS.}

To summarize, by exploiting the ability to optically control free-carrier concentrations in ITO 0D nanocrystals, we drive a significant injection of holes into $1 \mathrm{~L}-\mathrm{MoS}_{2}$ that is robust and stable over quasi-permanent timescales. This prototype 0D-2D hybrid structure has been demonstrated to optically transfer charges to values comparable to electrostatic doping, with an average light-driven metastable transfer of five carriers per ITO nanocrystal. By assuming a capacitor-like charging behavior, we estimate that capacitances in the $\mathrm{fF}$ range can be reached. This corresponds to stored energy and power densities in the range of $\mu \mathrm{J} \mathrm{cm}^{-2}$ and nW cm${ }^{-2}$, respectively. Our results suggest this novel nanosystem as a promising precursor for the further development into a fully light-driven 0D-2D hybrid nanocapacitor device.

\section{SUPPORTING INFORMATION AVAILABLE.}

Illustration of photodoping of ITO nanocrystals in solution, the permanent photodoping and the effect of photodoping on the single $\mathrm{MoS}_{2}$ layer without ITO nanocrystals. Fitting function of the temporal variation of the photoluminescence and estimation of the refractive index. This material is available free of charge via the Internet at http://pubs.acs.org.

\section{ACKNOWLEDGEMENTS.}

This project has received funding from the European Union's Horizon 2020 research and innovation programme under the Marie Skłodowska-Curie Grant Agreement No. [705444] (MOPTOPus) as well as Grant Agreement No. [734690] (SONAR), from the Graphene Core 2 program with Grant Agreement No. [785219GrapheneCore2] and from the European Research Council Grant Agreement No. [850875] (LightDYNAMO).Work at the Molecular Foundry was supported by the Office of Science, Office of Basic Energy Sciences, of the U.S. Department of Energy under Contract No. DE-AC02-05CH11231. 


\section{REFERENCES.}

(1) Kyeremateng, N. A.; Brousse, T.; Pech, D. Microsupercapacitors as Miniaturized Energy-Storage Components for on-Chip Electronics. Nat. Nanotechnol. 2017, 12 (1), 7. https://doi.org/10.1038/nnano.2016.196.

(2) Liang, J.; Mondal, A. K.; Wang, D.-W.; Iacopi, F. Graphene-Based Planar Microsupercapacitors: Recent Advances and Future Challenges. Adv. Mater. Technol. 2019, 4 (1), 1800200. https://doi.org/10.1002/admt.201800200.

(3) Zhang, P.; Wang, F.; Yu, M.; Zhuang, X.; Feng, X. Two-Dimensional Materials for Miniaturized Energy Storage Devices: From Individual Devices to Smart Integrated Systems. Chem. Soc. Rev. 2018, 47 (19), 7426-7451. https://doi.org/10.1039/C8CS00561C.

(4) Da, Y.; Liu, J.; Zhou, L.; Zhu, X.; Chen, X.; Fu, L. Engineering 2D Architectures toward HighPerformance Micro-Supercapacitors. Adv. Mater. 2019, 31 (1), 1802793. https://doi.org/10.1002/adma.201802793.

(5) Beidaghi, M.; Gogotsi, Y. Capacitive Energy Storage in Micro-Scale Devices: Recent Advances in Design and Fabrication of Micro-Supercapacitors. Energy Environ. Sci. 2014, 7 (3), 867-884. https://doi.org/10.1039/C3EE43526A.

(6) Wang, Z. L. Self-Powered Nanosensors and Nanosystems. Adv. Mater. 2012, 24 (2), 280-285. https://doi.org/10.1002/adma.201102958.

(7) Schimpf, A. M.; Lounis, S. D.; Runnerstrom, E. L.; Milliron, D. J.; Gamelin, D. R. Redox Chemistries and Plasmon Energies of Photodoped In2O3 and Sn-Doped In2O3 (ITO) Nanocrystals. $J$. Am. Chem. Soc. 2015, 137 (1), 518-524. https://doi.org/10.1021/ja5116953.

(8) Brozek, C. K.; Zhou, D.; Liu, H.; Li, X.; Kittilstved, K. R.; Gamelin, D. R. Soluble Supercapacitors: Large and Reversible Charge Storage in Colloidal Iron-Doped ZnO Nanocrystals. Nano Lett. 2018, 18 (5), 3297-3302. https://doi.org/10.1021/acs.nanolett.8b01264.

(9) Brozek, C. K.; Hartstein, K. H.; Gamelin, D. R. Potentiometric Titrations for Measuring the Capacitance of Colloidal Photodoped ZnO Nanocrystals. J. Am. Chem. Soc. 2016, 138 (33), 1060510610. https://doi.org/10.1021/jacs.6b05848.

(10) Valdez, C. N.; Delley, M. F.; Mayer, J. M. Cation Effects on the Reduction of Colloidal ZnO Nanocrystals. J. Am. Chem. Soc. 2018, 140 (28), 8924-8933. https://doi.org/10.1021/jacs.8b05144.

(11) Kriegel, I.; Urso, C.; Viola, D.; De Trizio, L.; Scotognella, F.; Cerullo, G.; Manna, L. Ultrafast Photodoping and Plasmon Dynamics in Fluorine-Indium Codoped Cadmium Oxide Nanocrystals for All-Optical Signal Manipulation at Optical Communication Wavelengths. J. Phys. Chem. Lett. 2016, 7 (19), 3873-3881. https://doi.org/10.1021/acs.jpclett.6b01904.

(12) Kriegel, I.; Scotognella, F.; Manna, L. Plasmonic Doped Semiconductor Nanocrystals: Properties, Fabrication, Applications and Perspectives. Phys. Rep. 2017, 674, 1-52. https://doi.org/10.1016/j.physrep.2017.01.003.

(13) Cohn, A. W.; Janßen, N.; Mayer, J. M.; Gamelin, D. R. Photocharging ZnO Nanocrystals: Picosecond Hole Capture, Electron Accumulation, and Auger Recombination. J. Phys. Chem. C 2012, 116 (38), 20633-20642. https://doi.org/10.1021/jp3075942.

(14) Valdez, C. N.; Braten, M.; Soria, A.; Gamelin, D. R.; Mayer, J. M. Effect of Protons on the Redox Chemistry of Colloidal Zinc Oxide Nanocrystals. J. Am. Chem. Soc. 2013, 135 (23), 8492-8495. https://doi.org/10.1021/ja4035945.

(15) Salihoglu, O.; Kakenov, N.; Balci, O.; Balci, S.; Kocabas, C. Graphene-Quantum Dot Hybrid Optoelectronics at Visible Wavelengths. ACS Photonics 2018, 5 (6), 2384-2390.

https://doi.org/10.1021/acsphotonics.8b00163.

(16) Goodman, A. J.; Dahod, N. S.; Tisdale, W. A. Ultrafast Charge Transfer at a Quantum Dot/2D Materials Interface Probed by Second Harmonic Generation. J. Phys. Chem. Lett. 2018, 9, 42274232. https://doi.org/10.1021/acs.jpclett.8b01606.

(17) Raja, A.; Montoya-Castillo, A.; Zultak, J.; Zhang, X.-X.; Ye, Z.; Roquelet, C.; Chenet, D. A.; van der Zande, A. M.; Huang, P.; Jockusch, S.; et al. Energy Transfer from Quantum Dots to Graphene and MoS2: The Role of Absorption and Screening in Two-Dimensional Materials. Nano Lett. 2016, 16 (4), 2328-2333. https://doi.org/10.1021/acs.nanolett.5b05012.

(18) Mak, K. F.; He, K.; Lee, C.; Lee, G. H.; Hone, J.; Heinz, T. F.; Shan, J. Tightly Bound Trions in Monolayer $\mathrm{MoS}_{2}$. Nat. Mater. 2013, 12 (3), 207-211. https://doi.org/10.1038/nmat3505. 
(19) Ross, J. S.; Wu, S.; Yu, H.; Ghimire, N. J.; Jones, A. M.; Aivazian, G.; Yan, J.; Mandrus, D. G.; Xiao, D.; Yao, W.; et al. Electrical Control of Neutral and Charged Excitons in a Monolayer Semiconductor. Nat. Commun. 2013, 4, 1474. https://doi.org/10.1038/ncomms2498.

(20) Yao, K.; Yan, A.; Kahn, S.; Suslu, A.; Liang, Y.; Barnard, E. S.; Tongay, S.; Zettl, A.; Borys, N. J.; Schuck, P. J. Optically Discriminating Carrier-Induced Quasiparticle Band Gap and Exciton Energy Renormalization in Monolayer MoS2. Phys. Rev. Lett. 2017, 119 (8), 087401. https://doi.org/10.1103/PhysRevLett.119.087401.

(21) Ilavsky, J.; Jemian, P. R. Irena: Tool Suite for Modeling and Analysis of Small-Angle Scattering. $J$. Appl. Crystallogr. 2009, 42 (2), 347-353. https://doi.org/10.1107/S0021889809002222.

(22) Zhang, K.; Borys, N. J.; Bersch, B. M.; Bhimanapati, G. R.; Xu, K.; Wang, B.; Wang, K.; Labella, M.; Williams, T. A.; Haque, M. A.; et al. Deconvoluting the Photonic and Electronic Response of 2D Materials: The Case of MoS2. Sci. Rep. 2017, 7 (1), 16938. https://doi.org/10.1038/s41598-01716970-6.

(23) Durham, D. B.; Ogletree, D. F.; Barnard, E. S. Scanning Auger Spectromicroscopy Using the ScopeFoundry Software Platform. Surf. Interface Anal. 2018, 50 (11), 1174-1179. https://doi.org/10.1002/sia.6401.

(24) Crockett, B. M.; Jansons, A. W.; Koskela, K. M.; Johnson, D. W.; Hutchison, J. E. Radial Dopant Placement for Tuning Plasmonic Properties in Metal Oxide Nanocrystals. ACS Nano 2017, 11 (8), 7719-7728. https://doi.org/10.1021/acsnano.7b01053.

(25) Jansons, A. W.; Hutchison, J. E. Continuous Growth of Metal Oxide Nanocrystals: Enhanced Control of Nanocrystal Size and Radial Dopant Distribution. ACS Nano 2016, 10 (7), 6942-6951. https://doi.org/10.1021/acsnano.6b02796.

(26) Jansons, A. W.; Plummer, L. K.; Hutchison, J. E. Living Nanocrystals. Chem. Mater. 2017, 29 (13), 5415-5425. https://doi.org/10.1021/acs.chemmater.7b00899.

(27) Li, Z.; Ye, R.; Feng, R.; Kang, Y.; Zhu, X.; Tour, J. M.; Fang, Z. Graphene Quantum Dots Doping of MoS2 Monolayers. Adv. Mater. 2015, 27 (35), 5235-5240. https://doi.org/10.1002/adma.201501888.

(28) Mouri, S.; Miyauchi, Y.; Matsuda, K. Tunable Photoluminescence of Monolayer MoS2 via Chemical Doping. Nano Lett. 2013, 13 (12), 5944-5948. https://doi.org/10.1021/nl403036h.

(29) Bao, W.; Borys, N. J.; Ko, C.; Suh, J.; Fan, W.; Thron, A.; Zhang, Y.; Buyanin, A.; Zhang, J.; Cabrini, S.; et al. Visualizing Nanoscale Excitonic Relaxation Properties of Disordered Edges and Grain Boundaries in Monolayer Molybdenum Disulfide. Nat. Commun. 2015, 6, 7993. https://doi.org/10.1038/ncomms8993.

(30) Borys, N. J.; Barnard, E. S.; Gao, S.; Yao, K.; Bao, W.; Buyanin, A.; Zhang, Y.; Tongay, S.; Ko, C.; Suh, J.; et al. Anomalous Above-Gap Photoexcitations and Optical Signatures of Localized Charge Puddles in Monolayer Molybdenum Disulfide. ACS Nano 2017, 11 (2), 2115-2123. https://doi.org/10.1021/acsnano.6b08278.

(31) Kastl, C.; Koch, R. J.; Chen, C. T.; Eichhorn, J.; Ulstrup, S.; Bostwick, A.; Jozwiak, C.; Kuykendall, T. R.; Borys, N. J.; Toma, F. M.; et al. Effects of Defects on Band Structure and Excitons in WS2 Revealed by Nanoscale Photoemission Spectroscopy. ACS Nano 2019. https://doi.org/10.1021/acsnano.8b06574.

(32) Kossacki, P. Optical Studies of Charged Excitons in II-VI Semiconductor Quantum Wells. J. Phys. Condens. Matter 2003, 15 (13), R471. https://doi.org/10.1088/0953-8984/15/13/201.

(33) Siviniant, J.; Scalbert, D.; Kavokin, A. V.; Coquillat, D.; Lascaray, J.-P. Chemical Equilibrium between Excitons, Electrons, and Negatively Charged Excitons in Semiconductor Quantum Wells. Phys. Rev. B 1999, 59 (3), 1602-1604. https://doi.org/10.1103/PhysRevB.59.1602.

(34) Chernikov, A.; van der Zande, A. M.; Hill, H. M.; Rigosi, A. F.; Velauthapillai, A.; Hone, J.; Heinz, T. F. Electrical Tuning of Exciton Binding Energies in Monolayer WS2. Phys. Rev. Lett. 2015, 115 (12), 126802. https://doi.org/10.1103/PhysRevLett.115.126802.

(35) Wang, J.; Ardelean, J.; Bai, Y.; Steinhoff, A.; Florian, M.; Jahnke, F.; Xu, X.; Kira, M.; Hone, J.; Zhu, X.-Y. Optical Generation of High Carrier Densities in 2D Semiconductor Heterobilayers. Sci. $A d v$. 2019, 5 (9), eaax0145. https://doi.org/10.1126/sciadv.aax0145.

(36) Geva, N.; Shepherd, J. J.; Nienhaus, L.; Bawendi, M. G.; Van Voorhis, T. Morphology of Passivating Organic Ligands around a Nanocrystal. J. Phys. Chem. C 2018, 122 (45), 26267-26274. https://doi.org/10.1021/acs.jpcc.8b08413. 
(37) Minami, T.; Miyata, T.; Yamamoto, T. Work Function of Transparent Conducting Multicomponent Oxide Thin Films Prepared by Magnetron Sputtering. Surf. Coat. Technol. 1998, 108-109, 583-587. https://doi.org/10.1016/S0257-8972(98)00592-1.

(38) Kang, J.; Tongay, S.; Zhou, J.; Li, J.; Wu, J. Band Offsets and Heterostructures of Two-Dimensional Semiconductors. Appl. Phys. Lett. 2013, 102 (1), 012111. https://doi.org/10.1063/1.4774090.

(39) Wang, Ch. Y.; Cimalla, V.; Kups, Th.; Röhlig, C.-C.; Romanus, H.; Lebedev, V.; Pezoldt, J.; Stauden, Th.; Ambacher, O. Photoreduction and Oxidation Behavior of In2O3 Nanoparticles by Metal Organic Chemical Vapor Deposition. J. Appl. Phys. 2007, 102 (4), 044310. https://doi.org/10.1063/1.2770831.

(40) Wang, N.; Niu, W.; Li, L.; Liu, J.; Tang, Z.; Zhou, W.; Chen, S. Oxygen Electroreduction Promoted by Quasi Oxygen Vacancies in Metal Oxide Nanoparticles Prepared by Photoinduced Chlorine Doping. Chem. Commun. 2015, 51 (53), 10620-10623. https://doi.org/10.1039/C5CC02808F.

(41) Agrawal, A.; Kriegel, I.; Runnerstrom, E. L.; Scotognella, F.; Llordes, A.; Milliron, D. J. Rationalizing the Impact of Surface Depletion on Electrochemical Modulation of Plasmon Resonance Absorption in Metal Oxide Nanocrystals. ACS Photonics 2018, 5 (5), 2044-2050. https://doi.org/10.1021/acsphotonics.7b01587.

(42) Zandi, O.; Agrawal, A.; Shearer, A. B.; Reimnitz, L. C.; Dahlman, C. J.; Staller, C. M.; Milliron, D. J. Impacts of Surface Depletion on the Plasmonic Properties of Doped Semiconductor Nanocrystals. Nat. Mater. 2018, 17 (8), 710-717. https://doi.org/10.1038/s41563-018-0130-5. 
TABLE OF CONTENT (TOC)

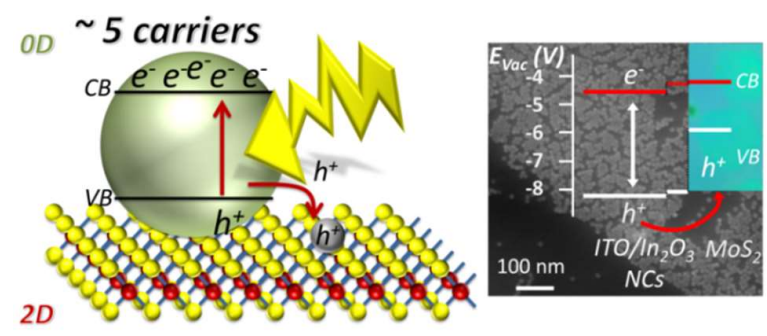

